\title{
PARTIAL RESECTION OF THE DISCOID MENISCUS IN THE CHILD'S KNEE
}

\author{
KYOSUKE FUJIKAWA, FUJIO ISEKI, YUETSU MIKURA \\ From the Department of Orthopaedic Surgery, Keio University, Tokyo
}

\begin{abstract}
Seven children underwent partial meniscectomy for symptomatic discoid lateral meniscus. This procedure, modifying the discoid meniscus to the normal semilunar shape, was indicated only when degeneration or tear was minimal, when the meniscus was not abnormally thickened nor of Wrisberg type, when it was not hypermobile, when the capsular attachment was intact, and when the residual meniscus was free from abnormality.

The results were excellent clinically, radiologically and arthroscopically, rehabilitation was shortened to half that required for total meniscectomy and the residual meniscus functioned entirely normally.
\end{abstract}

In the past, abnormalities of the meniscus in children have given difficulties in diagnosis because of the ambiguity of the patient's complaints, the uncertainty of clinical signs and the inadequate information provided by plain radiographs. Precise diagnosis has become possible by means of double-contrast arthrography and arthroscopy.

The majority of meniscal problems in children are due to a discoid lateral meniscus and this is the main difference between children and adults. The initial management of these problems in children should be conservative, with operation reserved for those patients who do not improve.

Recent biomechanical studies of knee function have revealed the importance of the menisci, and partial resection of the torn meniscus in adults has been advised (Cargill and Jackson 1976; McGinty, Geuss and Marvin 1977; Iseki and Fujikawa 1978). This paper reports our experience in performing partial resection of the discoid meniscus in children, with excellent results; it includes the indications for operation, the technique and a discussion of the advantages.

\section{CLINICAL MATERIAL}

Over the past six years, we have operated on 64 knees in children for internal derangement. Meniscectomy was performed in 39 of these joints, of which 32 were for discoid lateral meniscus, four for damage to a normal semilunar lateral meniscus and three for damage to a normal medial meniscus. The youngest patient was a three-year-old boy, and numbers of patients increased in direct proportion to age (Fig. 1). All patients under the age of 14 had discoid menisci, and in all but one case the lateral menisci of both knees were discoid in shape. In these patients with symptoms secondary to discoid

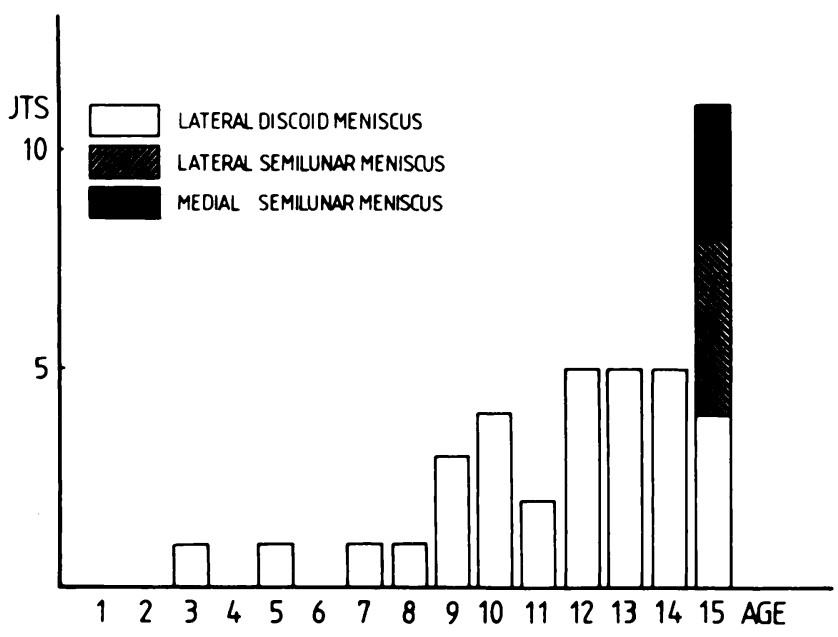

Fig. 1

Age distribution of children who underwent meniscectomy.

menisci, we performed operation on one side in 24 children and on both knees in four children. This confirms our previously reported observation on arthrographic and arthroscopic study that the meniscus is almost always of the same shape in right and left knees. This was so in 98.7 per cent of patients on the lateral side 
and 99.3 per cent of patients on the medial side (Fujikawa et al. 1978). The present series confirms that there are many clinically silent discoid menisci in children (Fig. 2).

Recently, total resection has been the recom-

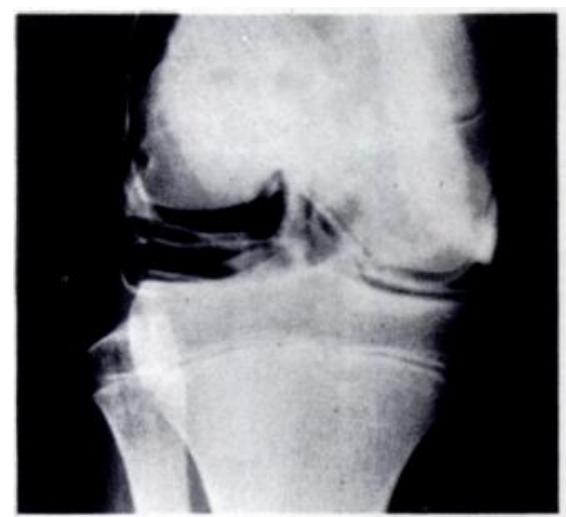

Fig. 2

Arthrogram of a clinically silent discoid meniscus in a child. There are no degenerative changes on the meniscus nor on the articular cartilages.

mended treatment for symptomatic discoid menisci only when conservative methods of treatment, such as rest, short-term immobilisation in plaster and quadriceps drill, have failed. Surgical treatment has been restricted to those patients with repeated or persistent locking, a block to extension or severe pain. In these cases, double-contrast arthrography or arthroscopy may show rapid secondary degenerative changes of the articular cartilage and bone, especially of the tibial surface, demonstrating the adverse effect of the symptomatic discoid meniscus on the joint. Excellent clinical results have been obtained in over 95 per cent of our patients who have undergone total resection, but some degenerative changes of the joint surfaces are unavoidable.

For the past few years we have undertaken partial resection of the free edge of the discoid meniscus, modifying it to the normal semilunar shape in order to minimise the effect of meniscectomy.

\section{PARTIAL RESECTION OF THE DISCOID MENISCUS}

Indications. This procedure is indicated when the discoid meniscus shows only slight degeneration or a minimal tear; when it is not abnormally thickened nor of the Wrisberg type; when it is not hypermobile; and when the capsular attachment is intact. These conditions should be accurately confirmed before operation by double-contrast arthrography and arthroscopy.

Operative procedure. This is carried out via a fourcentimetre anterolateral incision below a pneumatic tourniquet with the knee flexed to 90 degrees over the end of the table. No abnormality is usually apparent on the femoral surface of the discoid meniscus, but slight degeneration or a minimal tear may be seen on its tibial surface or within its structure. Fingertip palpation of the femoral surface of the discoid meniscus, moving from the capsular side to the free edge, may locate a slight sagittal irregularity. The meniscus is incised along the line of this irregularity using a small knife and scissors in as clean a manner as possible. The anterior insertion on the meniscus is left untouched, and by pulling this anterior part towards the intercondylar fossa, it is possible to complete partial resection of the central and posterior parts of the meniscus (Fig. 3). The posterior insertion of the meniscus is also left intact. The resected portion of meniscus is almost circular in shape (Fig. 4).

It is then necessary to confirm that the residual rim of meniscus is free from any degeneration or tear. The residual meniscus may be inspected by inserting an arthroscope through the incision to observe closely the posterior edge of the remaining rim. If degeneration or tearing is observed, total resection should be performed.
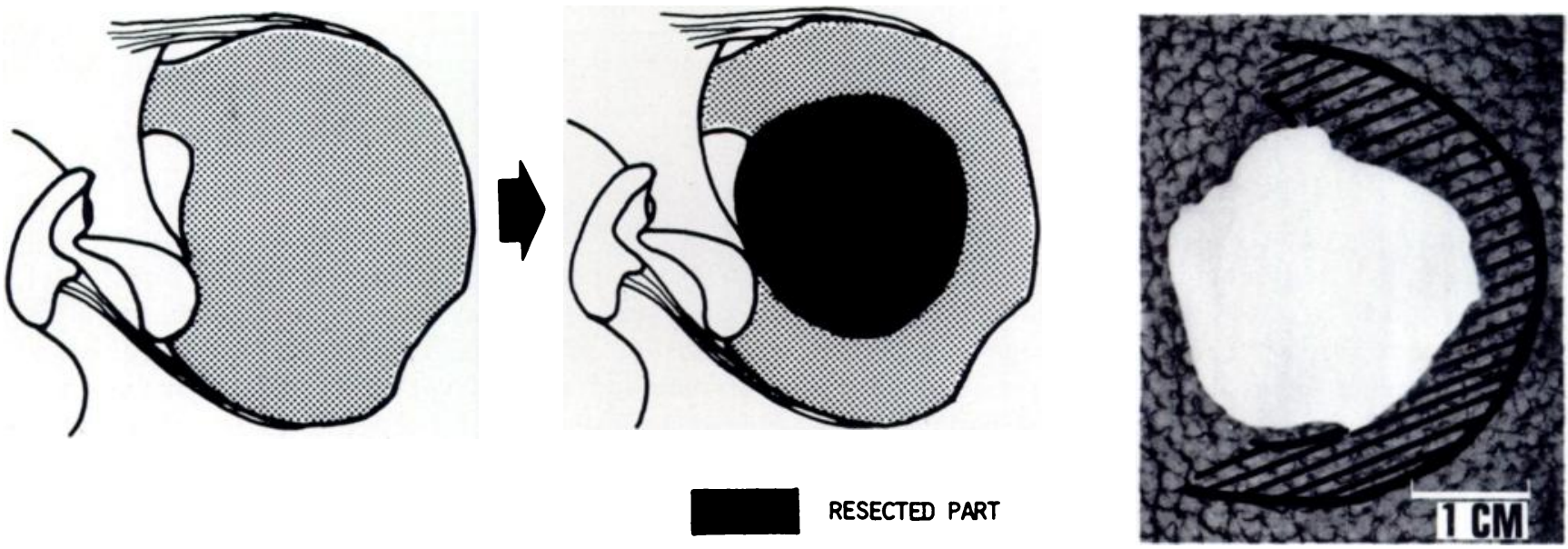

Fig. 3

Fig. 4

Figure 3-Diagrams to show partial resection of the discoid meniscus. Figure 4-Resected part of the discoid meniscus (Case 1 ). It was almost round in shape. 
After-care. On the day after operation quadriceps drill and active exercises are begun. A full range of movement should be achieved within a week or so, but if extension has been blocked for a considerable time before operation it will take longer to regain full extension, and intermittent traction may be required in combination with the other exercises. The period of rehabilitation is only half that required for total meniscectomy.

\section{CASE REPORTS}

Partial meniscectomy for discoid meniscus was undertaken in seven children. Patients in whom partial resection was carried out for flap or longitudinal tears which extended to the femoral surface have not been included in the series.

Case 1. A nine-year-old boy had suffered pain in the left knee for several years. One month before he was seen the knee had deteriorated after a fall. Swelling had settled but on examination he had a 20-degree flexion deformity, pain and a limp with marked atrophy of the quadriceps. The rotatory friction test (Smillie 1970) was

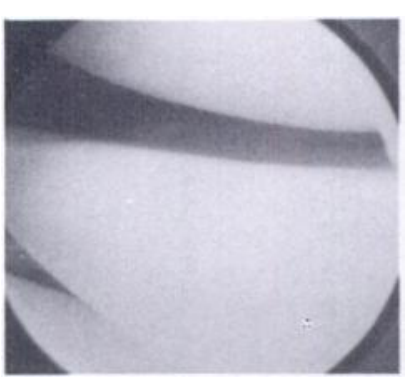

Fig. 5

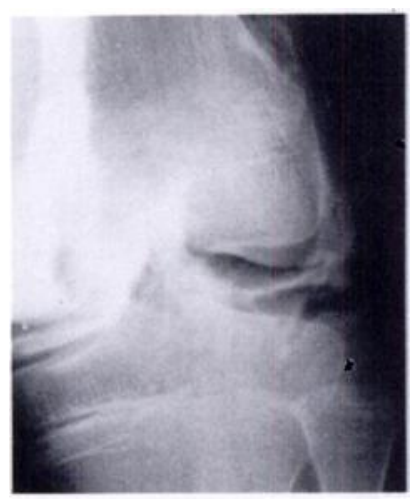

Fig. 6

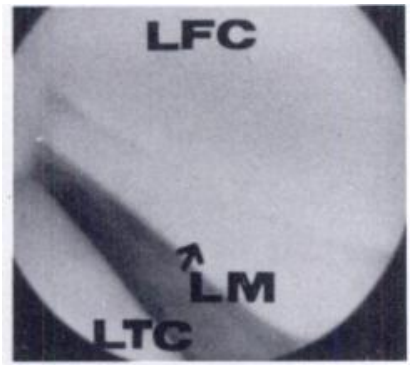

Fig. 7

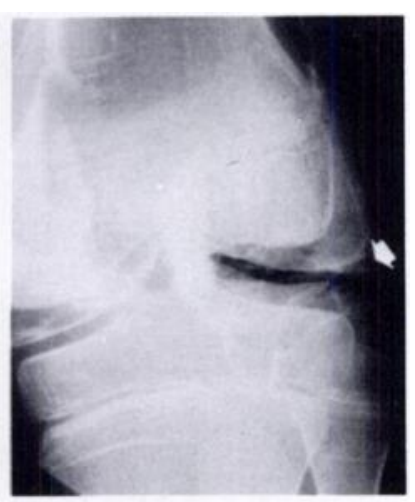

Fig. 8
Case 1. Figures 5 and 6 -Arthroscopy and arthrography before operation. Figures 7 and 8-After operation, both pictures show the discoid meniscus modified to semilunar shape (arrowed) with no degenerative changes (LFC, lateral femoral condyle; LTC lateral tibial condyle; LM lateral meniscus).

positive on the lateral side. Flexion was almost full but forcing this produced severe pain in the lateral joint space. Plain radiographs were normal, but arthrography revealed completely discoid lateral menisci in both knees. There was no evidence of tear or of degeneration in the clinically normal right knee, but on the left there was slight degeneration and a minimal tear on the tibial surface of the meniscus, while pooling of the contrast medium on the articular cartilage indicated slight degenerative change. These findings were confirmed by arthroscopy which also excluded a Wrisberg-type of meniscus or hypermobility (Figs 5 to 8 ).
At operation the symptomatic discoid meniscus was partially resected, thereby modifying it to the normal semilunar shape. One year later double-contrast arthrography showed the residual meniscus to be almost normal in shape and without any degeneration or tear. Arthroscopy showed a smooth free margin in a meniscus which was normal in size, and no further degenerative changes of the articular cartilage. Two years after operation the results were excellent on clinical and radiological examination.

Case 2. A 13-year-old girl had been complaining of pain in both knees, worse on the right, for about six months. For two months she had been unable to extend the right knee fully and had limped. On examination the right knee showed a 20-degree flexion deformity but no effusion. The rotatory friction test was positive at 100 degrees on the lateral side. Plain radiography showed slight sclerosis of the subchondral bone of the lateral tibial condyle. Arthrography and arthroscopy showed a completely discoid lateral meniscus and confirmed degenerative changes on the tibial articular surface (Figs 9 to 12).

The knee did not settle with conservative treatment and repeat arthrography after two months showed deterioration of the degenerative changes. Partial resection of the discoid meniscus was carried out. An arthroscope was used at operation to inspect the posterior part of the residual meniscus and this revealed only slight degenerative changes.

The result a year and a half later was excellent and plain radiographs showed no progression of the osteoarthritic changes. Arthrography confirmed slight degenerative changes on the free edge of the residual meniscus. These findings were confirmed on arthroscopy but were confined to the posterior segment.

Case 3. A seven-year-old boy sprained his left knee on landing from a vaulting horse and gave a one-year history of previous slight pain. The knee had not improved with three months of conservative management, and the child still had a block to extension and atrophy of the muscles. On examination the knee was flexed to 20 degrees with some thickening of the lateral infrapatellar fat pad but no effusion. The rotatory friction test was positive at 40 degrees and there was tenderness over the front of the meniscus but no abnormal click was

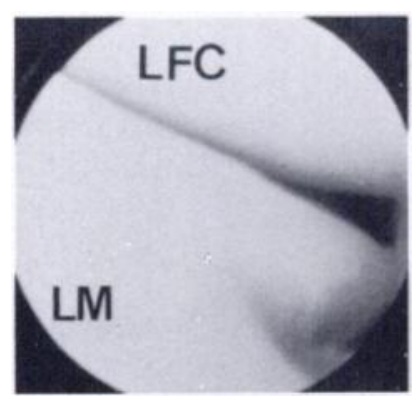

Fig. 9

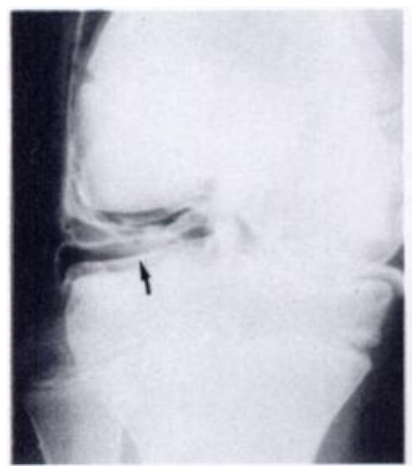

Fig. 10

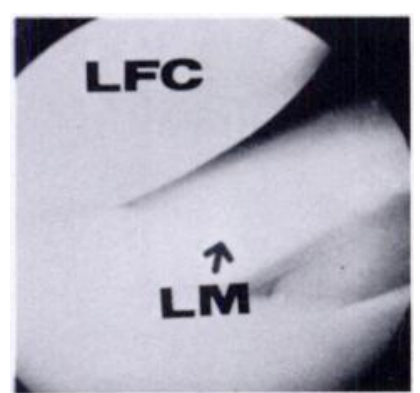

Fig. 11

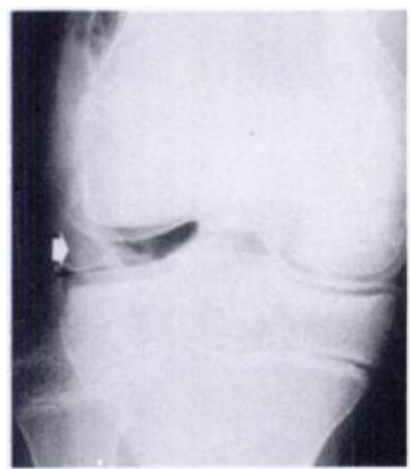

Fig. 12
Case 2. Figures 9 and 10-Arthroscopy and arthrography in a 13-year-old girl before operation. Figures 11 and $12-\mathrm{A}$ year and a half after operation slight degenerative change on the posterior segment of the modified meniscus could be seen. 
found. Plain radiographs were normal but arthrography showed a discoid lateral meniscus with some irregularity of the anterior portion and hypertrophy of the infrapatellar fat pad. Arthroscopy showed an abnormally large anterior horn with congestion and some degeneration of the fat pad.

At operation the abnormally enlarged anterior horn was partially resected and a detachment of the anterior margin was carefully sutured to its original position. The hypertrophied fringe of the fat pad was also partially removed. After operation the flexion deformity gradually improved and 18 months later the clinical and radiological results were excellent.

\section{RESULTS}

The results of partial resection of the discoid meniscus by the technique described were excellent both clinically and radiologically in all seven children. Furthermore, the period of rehabilitation after this procedure was shortened to half of the time required after total meniscectomy except in those patients who had had a prolonged period with a block to extension. Doublecontrast arthrography and arthroscopy during follow-up have shown no progression of the degenerative changes present in the articular cartilage before operation. In one patient (Case 2 ) there was some slight degenerative change of the posterior segment of the residual meniscus which was presumed to exist before operation. This degeneration was clinically silent and it will be interesting in follow-up to assess possible development into osteoarthritis.

\section{DISCUSSION}

In children, total resection of a discoid meniscus is performed only when it is unavoidable, although it has been said that the prognosis after total meniscectomy is better in children than in adults (Kobayashi 1975). However, significant degenerative changes are inevitable after meniscectomy (Fairbank 1948; Jackson 1968; Kobayashi 1975; Cargill and Jackson 1976; McGinty et al. 1977).

Recent biomechanical research (Kettelkamp and Jacobs 1972; Maquet, Van de Berg and Simonet 1975; Walker and Erkman 1975; Seedhom 1979; Seedhom and Hargreaves 1979) has highlighted the importance of the meniscus in compensating for the incongruity between femur and tibia, in load transmission, in providing for rotation, as a shock absorber, in the provision of stability, in the distribution of joint pressures and in the spreading of synovial fluid. Partial resection or repair of peripheral tears of the meniscus have been performed in the adult in order to preserve these functions of the meniscus (Aarstrand 1954; Cargill and Jackson 1976; McGinty et al. 1977; Iseki and Fujikawa 1978).

Reduction of the contact areas between femur and tibia have been reported as a result of meniscectomy. Maquet et al. (1975) showed that the contact areas ranged from 20 square centimetres at full extension to 11 square centimetres at 90 degrees of flexion in the normal knee, and that these areas were reduced to 12 square centimetres and six square centimetres respectively after total meniscectomy; this means that the menisci form a large part of the weight-bearing surface of the knee in any position of flexion. Seedhom (1979) discussed the load-bearing function of the menisci and recommended that only the free edge of the meniscus should be resected. He stated that the intact meniscus transmits from 70 to 90 per cent of the total load across the joint, and that, after removal of a bucket-handle fragment, the residual rim still transmits a significant proportion of this load.

The experiments of Takao (1971) using mature rabbits and of Cox et al. (1975) on dogs showed that only slight degenerative change followed partial resection, whereas marked degenerative changes were seen as a result of total meniscectomy even if optimal regeneration was achieved. Takao concluded that partial meniscectomy is superior because the function of the meniscus is, to a large extent, preserved. Aarstrand (1954), McGinty et al. (1977) and Iseki and Fujikawa (1978) reported better subjective functional and

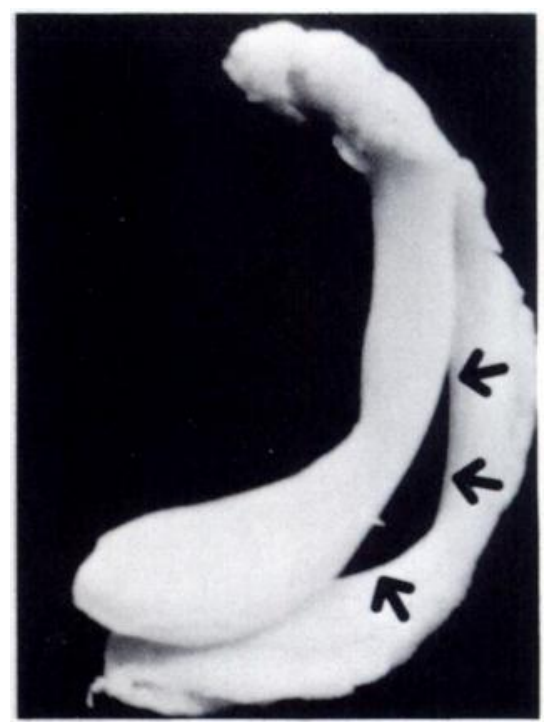

Fig. 13

A longitudinal tear of the meniscus, shows the torn edge to be as smooth as a normal free edge of a semilunar meniscus.

anatomical results from partial meniscectomy in adults, provided that strict indications were followed.

In children, we perform this partial resection of the free edge of the discoid meniscus, modifying it to the normal semilunar shape, for the following reasons: to allow the residual meniscus to function in a manner similar to that of the normal meniscus; to prevent some of the instability resulting from meniscectomy; to minimise the extent of operative intervention; and to shorten the rehabilitation period. Adaptation of the residual rim of meniscus may often be seen in adults after a longitudinal tear (Fig. 13). There are, however, some doubts concerning the long-term results of this 
procedure. The arrangement of collagen fibres is different in the semilunar and the discoid cartilage (Amako 1960) and it is possible that further tearing or degeneration of the residual meniscus could occur after operation. However, should the long-term results be as good as the early results, this technique will be advisable in the treatment of symptomatic discoid meniscus in children.

\section{REFERENCES}

Aarstrand T. Treatment of meniscal rupture of the knee joint. A follow-up examination of material where only the ruptured part of the meniscus has been removed. Acta Chir Scand 1954;107:146-57.

Amako T. On the injuries of the menisci in the knee joint of Japanese. J Jap Orthop Ass 1960;33:1289-1322.

Cargill AOR, Jackson JP. Bucket-handle tear of the medial meniscus. J Bone Joint Surg [Am] 1976;58-A:248-51.

Cox JS, Nye CE, Schaefer WW, Woodstein IJ. The degenerative effects of partial and total resection of the medial meniscus in dogs' knees. Clin Orthop 1975;109:178-83.

Fairbank TJ. Knee joint changes after meniscectomy. J Bone Joint Surg [Br] 1948;30-B:664-70.

Fujikawa K, Tomatsu T, Matsu K, Koiae A, Tanaka Y, Iseki F. Morphological analysis of meniscus and articular cartilage in the knee joint by means of arthrogram. J Jap Orthop Ass 1978;52:203-15.

Iseki F, Fujikawa K. Partial resection of the injured meniscus. XIV World Congress of SICOT, Kyoto, 1978.

Jackson JP. Degenerative changes in the knee after meniscectomy. Br Med J 1968;ii:525-7.

Kettelkamp DB, Jacobs AW. Tibiofemoral contact area-determination and implications. J Bone Joint Surg [Am] 1972;54-A:349-56.

Kobayashi A. Discoid meniscus of the knee joint. Clin Orthop Surg Jap 1975;10:10-24.

Maquet PG, Van de Berg AJ, Simonet JC. Femorotibial weight-bearing areas: experimental determination. J Bone Joint Surg [Am] $1975 ; 57-\mathbf{A}: 766-71$

McGinty JB, Geuss LF, Marvin RA. Partial or total meniscectomy. J Bone Joint Surg [Am] 1977;59-A:763-6.

Ritchie D. Meniscectomy in children. J Bone Joint Surg [Br] 1965;47-B:596.

Seedhom BB. Transmission of the load in the knee joint with special reference to the role of the menisci. Part I. Anatomy, analysis and apparatus. Eng Med 1979;8:207-19.

Seedhom BB, Hargreaves DJ. Transmission of the load in the knee joint with special reference to the role of the menisci. Part II. Experimental results, discussion and conclusion. Eng Med 1979;8:220-8.

Smillie IS. Injuries of the knee joint. 4th ed. Edinburgh and London: E \& S Livingstone, 1970: 77-9.

Takao T. An experimental study on the development of the osteoarthritis of the knee joint with special reference of the degree of resection of the meniscus. J Jap Orthop Ass 1971;45:731-42.

Walker PS, Erkman J. The role of the menisci in force transmission across the knee. Clin Orthop 1975;109:184-92. 